\title{
Rapid Ultrasensitive Gel-Free Immunoblotting with Magnetic Labels
}

Yuri M. Shlyapnikov*, Igor L. Kanev, and Elena A. Shlyapnikova

Institute of Theoretical and Experimental Biophysics, Russian Academy of Sciences, Pushchino, Moscow Region, 142290 Russia.

*Correspondence. E-mail: yuri.shlyapnikov@gmail.com Fax: 7-496-733-0553.

\section{Supporting Information}

Contents: measuring the diffusion coefficient; estimating the thickness of the gap between membranes; the effect of electroosmosis on protein mobility; possible explanations of the electrophoretic mobility discrepancies; smoothed cellulose membrane; measuring the efficiency of protein immobilization; comparing the sensitivity of different detection techniques; the sensitivity of traditional dot-blotting of IL-1 $\beta$; removing magnetic beads from the surface.

\section{Measuring the diffusion coefficient}

For diffusion measurements, after an electric field was turned off, the clamped membrane was incubated in a humid chamber, the images of blurring bands were taken under UV illumination at specific time intervals, and the increase in the width of the leading band was measured. As seen in Fig. S1, the linear slope is $0.34 \mathrm{~mm}^{2} / \mathrm{h}$, which corresponds to a diffusion coefficient $D=l^{2} / 2 t=$ $0.2 \mathrm{~mm}^{2} / \mathrm{h}=5 \times 10^{-7} \mathrm{~cm}^{2} / \mathrm{s}$. This value practically coincides with the published diffusion coefficient in the solution, $6 \times 10^{-7} \mathrm{~cm}^{2} / \mathrm{s}$ [S1]. The question is why the diffusion coefficient is the same as in the solution, whereas the electrophoretic mobility is two to three times lower, as discussed above. We believe that this is due to the small component of the electric field normal to the membrane surface, which is present during electrophoreses. It presses protein molecules to the membrane, where their interaction with the surface reduces electrophoretic mobility. At the same time, the diffusion in the absence of electric field takes place far from the surface.

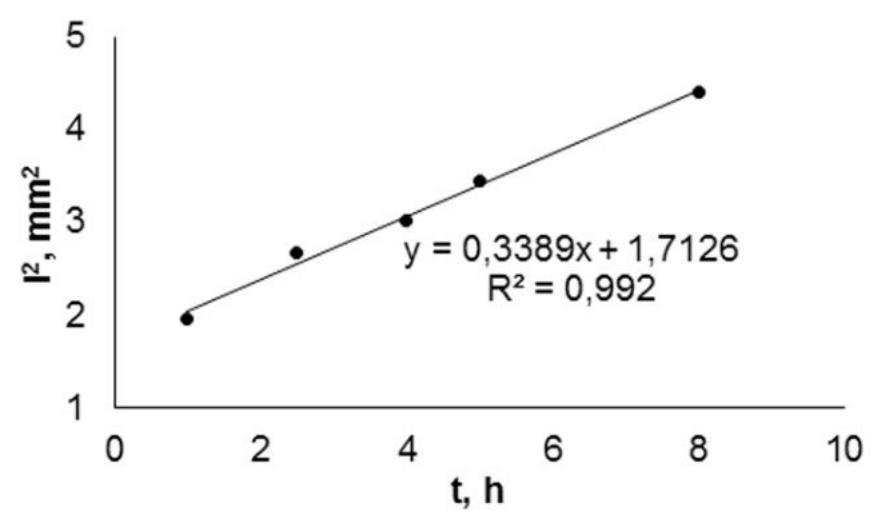

Fig. S1. Measurement of the diffusion constant of FITC-BSA on the surface of the cellulose membrane. The squared distance travelled by the protein is plotted against the diffusion time. 
Estimating the thickness of the gap between the membranes was carried out by two independent methods.

According to the first method, the FITC-BSA band area as a function of the protein quantity was estimated by loading the specified amount of FITC-BSA monomer into the sample loop (see Fig. 1 in the main text), running electrophoresis for $0.5 \mathrm{~min}$, and measuring the band area under UV illumination. As shown in Fig. S2A, at low protein amounts the band area remains unchanged, which is determined by factors that are not related to the limited capacity of the gap. At higher protein amounts, the band area increases linearly at the slope of by $20 \mathrm{~mm}^{2}$ per one $\mu \mathrm{g}$ of protein. Assuming that the volumetric concentration of the protein in the band is $\sim 10 \%$ (in order of magnitude, this is close to the solubility of the protein), this leads to a thickness of the gap of $\sim 1$ $\mu \mathrm{m}$ between the membrane sheets.

According to the second method, the electric conductance of a pair of touching membranes was measured. The conductance was measured along the membrane surface (Fig. S2B). The membranes and the gap can be considered as connected in a parallel circuit, and, thus, their total conductance is the sum of the membrane conductance and the conductance of the gap between them, which is filled with buffer. The measured conductivity of the Py-borate buffer was $182 \pm 5$ $\mu \mathrm{S} / \mathrm{cm}$. The resistance of $22.3 \times 11.0 \times 0.51 \mathrm{~mm}$, membrane measured along the membrane surface was $1.05 \pm 0.02 \mathrm{MOhm}$, corresponding to the conductivity of the swollen membrane $38 \pm 2 \mu \mathrm{S} / \mathrm{cm}$, which is $\sim 1 / 5$ of the buffer conductivity. To obtain the gap thickness, the conductance of a pair of touching swollen membranes was measured, as illustrated in Fig. S2B (excess buffer was preliminarily removed from the outer sides of the membranes with a filter paper). The membranes were then separated from each other, wiped with filter paper, and the total conductance of the two separated membranes was measured. The difference between these two values was attributed to the conductance of the gap filled with the buffer, from which its thickness was estimated using the buffer conductivity value presented above. The gap thickness was measured at various clamping forces (Fig. S2C), and it did not change in the range of 0-100 kPa, and only at higher forces a slight decrease was observed. We suppose that the membranes are pressed to each other by the Laplace pressure, which for $1 \mu \mathrm{m}$ meniscus radius is $p=2 \sigma / r=2 \times 72 \times 10^{-3} \mathrm{~J} / \mathrm{m}^{2} / 10^{-6} \mathrm{~m}=140 \mathrm{kPa}$. Thus, the membranes are already pressed by capillary forces, and the additional moderate clamping force does not affect the gap thickness. 

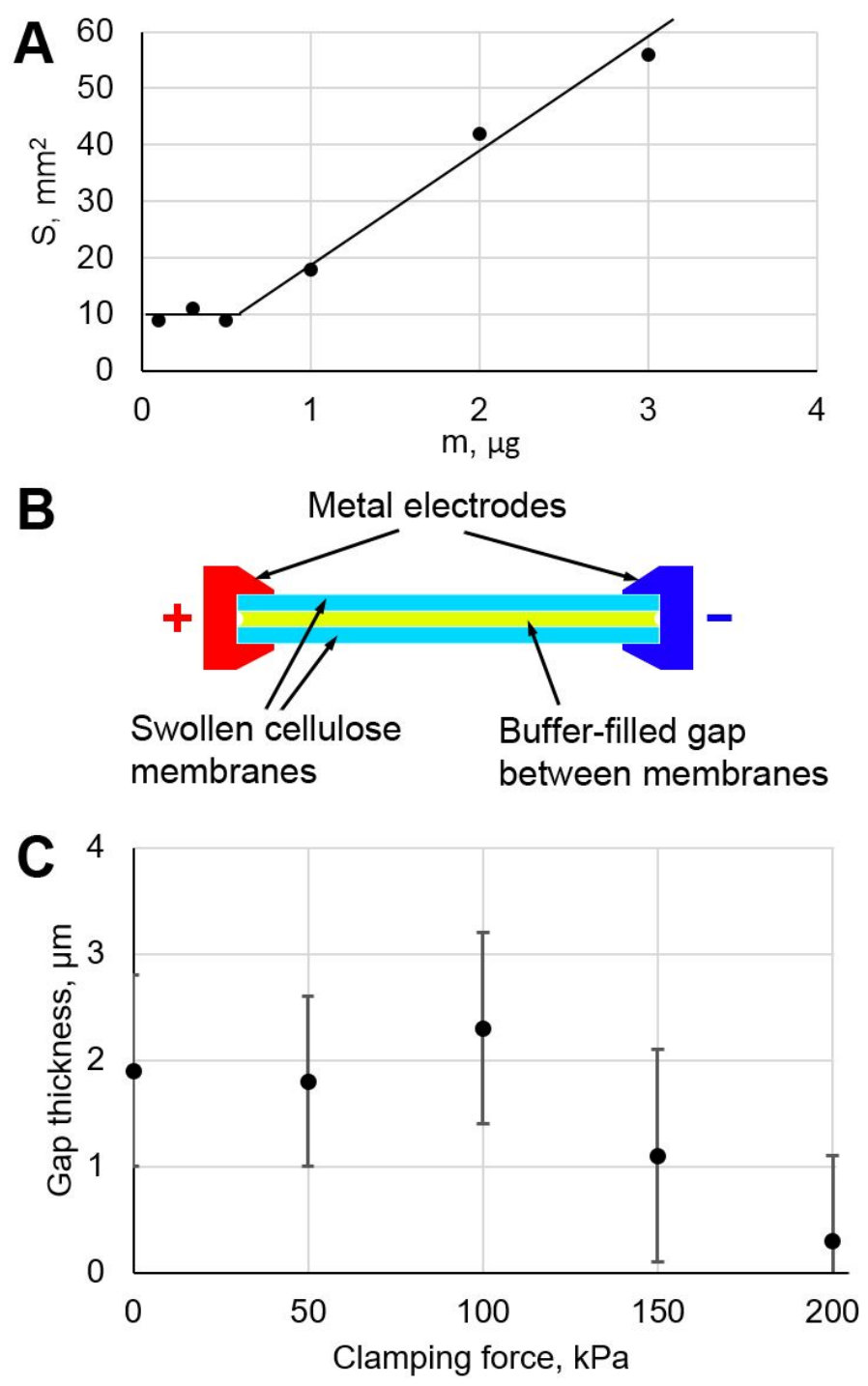

Fig. S2. (A) The FITC-BSA band area as a function of protein mass. (B) Schematic of the conductance measurement procedure. (C) The gap thickness calculated from the conductance data for various clamping forces. Error bars correspond to two standard deviations calculated from three independent measurements.

\section{The effect of electroosmosis on the protein mobility}

A positively charged membrane was obtained by immersing a smoothed dialysis membrane in a solution containing $100 \mathrm{mM}$ spermidine, $100 \mathrm{mM}$ EDC, $50 \mathrm{mM} \mathrm{MES,} \mathrm{pH} \mathrm{6.0,} \mathrm{for} 2 \mathrm{~h}$, followed by extensive washing with water. Figure S3 presents the electrophoretic mobility of the FITC-BSA monomer obtained in Py-borate buffer, $\mathrm{pH}$ 8.0, with a native (red) and a positively charged (green) membrane. In addition, the mobility in an acidic buffer containing $10 \mathrm{mM}$ glycine and $10 \mathrm{mM}$ acetic acid, $\mathrm{pH} 3.5$, with a reversed electrode polarity is presented (blue). According to published data [30], at such a $\mathrm{pH}$, BSA molecules have almost the same absolute charge value as at $\mathrm{pH} 8$, but with the opposite charge sign. Thus, the protein that is positively charged at $\mathrm{pH} 3.5$ moves toward the cathode at the same speed as the negatively charged BSA towards the anode. It 
is clear that the membrane charge and the polarity of electrodes have no effect on the protein mobility, and thus, the electroosmotic flow does not significantly contribute to mobility in our experimental conditions. If there was a significant electroosmotic flow, the direction of which could not be reversed by changing the $\mathrm{pH}$ from 8 to 3.5 (as we assume that the only ionizable groups in the cellulose membrane are carboxylic ones), it would result in a notable difference in the mobility of the positively and negatively charged protein. Thus, the observed mobility of proteins is purely electrophoretic, and no electroosmotic flow is detected, despite the fact that the membrane contains $\sim 10 \mathrm{mM}$ negative charges [23]. Probably, charges are distributed over the thickness of the membrane and are unable to create a significant $\zeta$-potential on its surface.

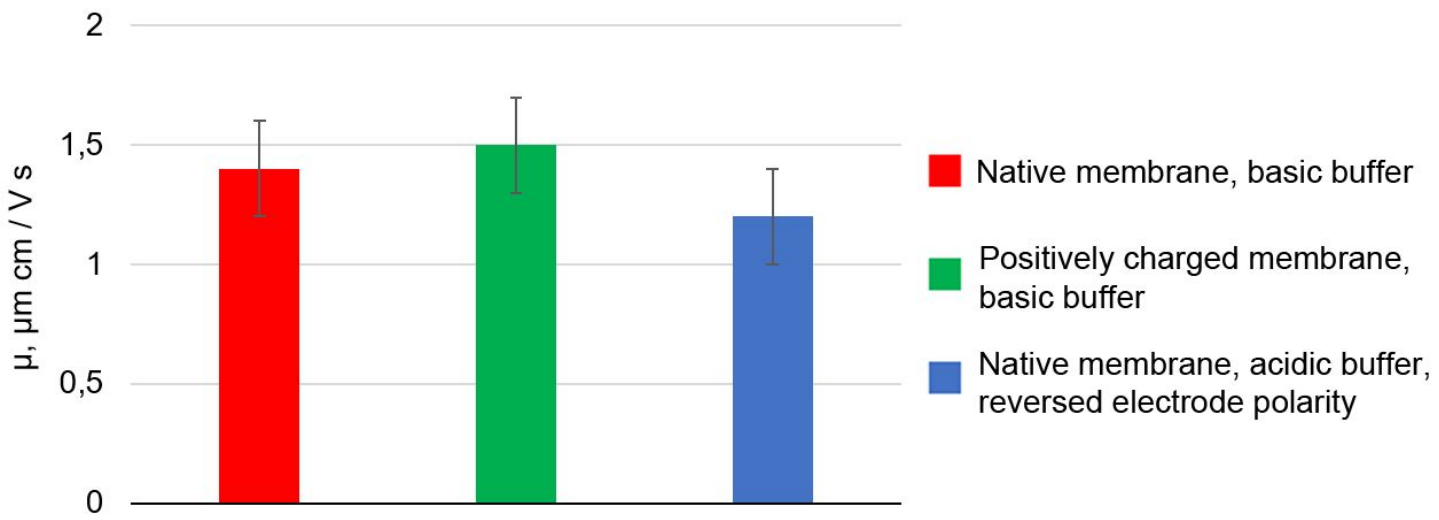

Fig. S3. The initial electrophoretic mobility with different polarity of electrodes and membrane charge. Error bars correspond to two standard deviations calculated from 5 independent experiments each. Student t-test with a confidence level of 0.95 was employed to conclude that the difference between the presented values is not statistically significant.

\section{Possible explanations of the electrophoretic mobility discrepancies}

As follows from Fig. 3B (black curves), when using the native commercial dialysis membrane, the protein mobility decreases during electrophoresis in a highly irreproducible manner. One possible explanation is based on irregularities in the membrane surface. If there is a small uncontrollable component of the electric field normal to the membrane surface, which is enabled by significant ionic conductivity of the swollen cellulose membrane (Supporting Information, p. 2), it pushes protein molecules into the pits on the rough membrane surface. Protein molecules become "trapped" in these irregularities and are unable to move further along the surface. An alternative hypothesis is that the distribution of the electric field in the gap creates potential grooves, followed by migrating proteins, and some of grooves are too tight to traverse. Both ideas were supported by AFM imaging of the native membrane (Fig. S5), which revealed pits and grooves with a depth of $\sim 100 \mathrm{~nm}$, which is significantly higher than the reported hydrodynamic radius of the BSA, $\sim 4 \mathrm{~nm}$ [30]. Similar effects were observed in a previous report [S2], where the electrophoresis of large (1-5 $\mu \mathrm{m})$ latex beads along a cellulose membrane was 
studied. The beads were hydrodynamically pressed to the surface, and their mobility strongly decreased upon an increase of the pressing force. The authors suggest that smoothing the surface is required to improve the separation efficiency. Therefore, we fabricated a membrane with a smooth surface by deposition of a "lacquering" cellulose layer, as described below, which was used in all further experiments.

A smoothed cellulose membrane was fabricated in accordance with the ideas outlined in the previous publication [S3]. A "lacquering" cellulose layer was deposited onto the membrane by precipitation from a $\mathrm{DMAc} / \mathrm{LiCl}$ solution (see experimental protocol below). To improve the adhesion to the "lacquering" layer, the membrane was pretreated with a diisocyanate crosslinker, which forms urethane bonds between the cellulose chains. Then, cellulose was precipitated, as illustrated in Fig. S4. Water diffuses through the dialysis membrane, causing the deposition of cellulose, whereas DMAc and lithium chloride are removed from the cellulose layer by diffusion into water. It was shown that the casting of cellulose films from the DMAc/LiCl solvent system leads to the formation of amorphous cellulose [S4], which is supposed to have lower adhesion than crystalline one. Low adhesion of the surface is essential for the immunodetection of proteins with magnetic beads (discussed below). Considering that $5 \mu \mathrm{L}$ of a $2 \%$ cellulose solution was used per $1 \mathrm{~cm}^{2}$ of the membrane area, an average thickness of the deposited layer is $\sim 1 \mu \mathrm{m}$, which is sufficient to fill pits and smooth out the irregularities of the native membrane surface. Though the precipitated cellulose layer is formed in contact with mica, it is much smoother than the native membrane, as confirmed by AFM data (Fig. S5 and Table S1). The observed granularity scale is 5-10 nm, which is comparable to the size of protein molecules. We expect that the granularity does not interfere with the electrophoretic movement of protein molecules along the surface. Although the procedure of membrane smoothing is rather complex, it does not have to be reproduced on a day-to-day basis. Instead, it could be performed once in large scale to simultaneously produce multiple membrane sheet, which could be further stored for at least several months (see Table S1).

An experimental protocol for smoothing of the dialysis membrane. A dialysis membrane sheet was soaked in water, glued to a steel O-ring (internal diameter $65 \mathrm{~mm}$ ) with a cyanoacrylate glue and dried overnight. Then, the membrane was treated with a solution of $1 \%$ toluene-2,4diisocyanate and 1\% trimethylamine in anhydrous acetonitrile for $1 \mathrm{~min}$, washed with acetonitrile, and dried in a stream of nitrogen. A $2 \%$ cellulose solution was obtained by dissolving pieces of the dialysis membrane in anhydrous N,N-dimethylacetamide (DMAc) containing 8\% (wt.) LiCl by vigorous stirring at room temperature. The cellulose solution was applied to the membrane ( 5 $\mu \mathrm{L}$ of solution per $1 \mathrm{~cm}^{2}$ ) and covered with a sheet of freshly cleaved mica. The reverse side of 
the membrane was then brought into contact with water, as illustrated in Fig. S4. After $14 \mathrm{~h}$ of incubation, the mica was carefully removed from the membrane, and the latter was rinsed with water and cut into strips of the appropriate size, dried in air and stored at room temperature for 160 days for future use in AFM, electrophoresis, and blotting experiments.

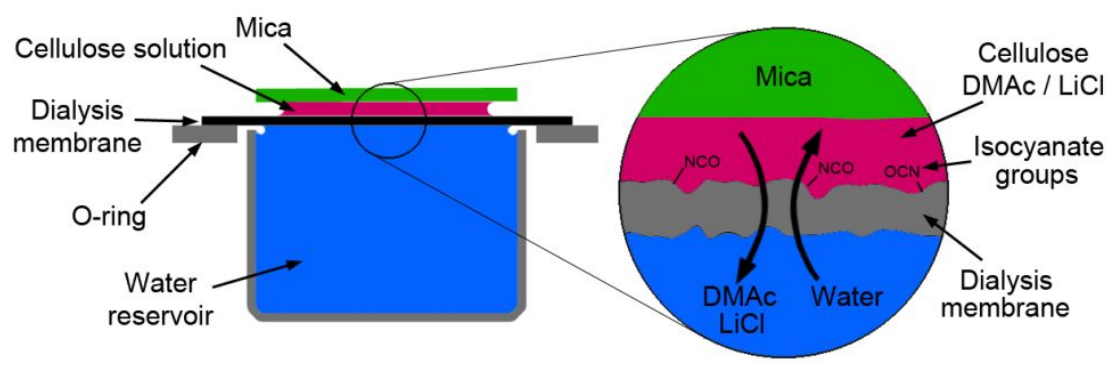

Fig S4. Schematic of the procedure of smoothing the surface of the dialysis membrane. See details in the text.

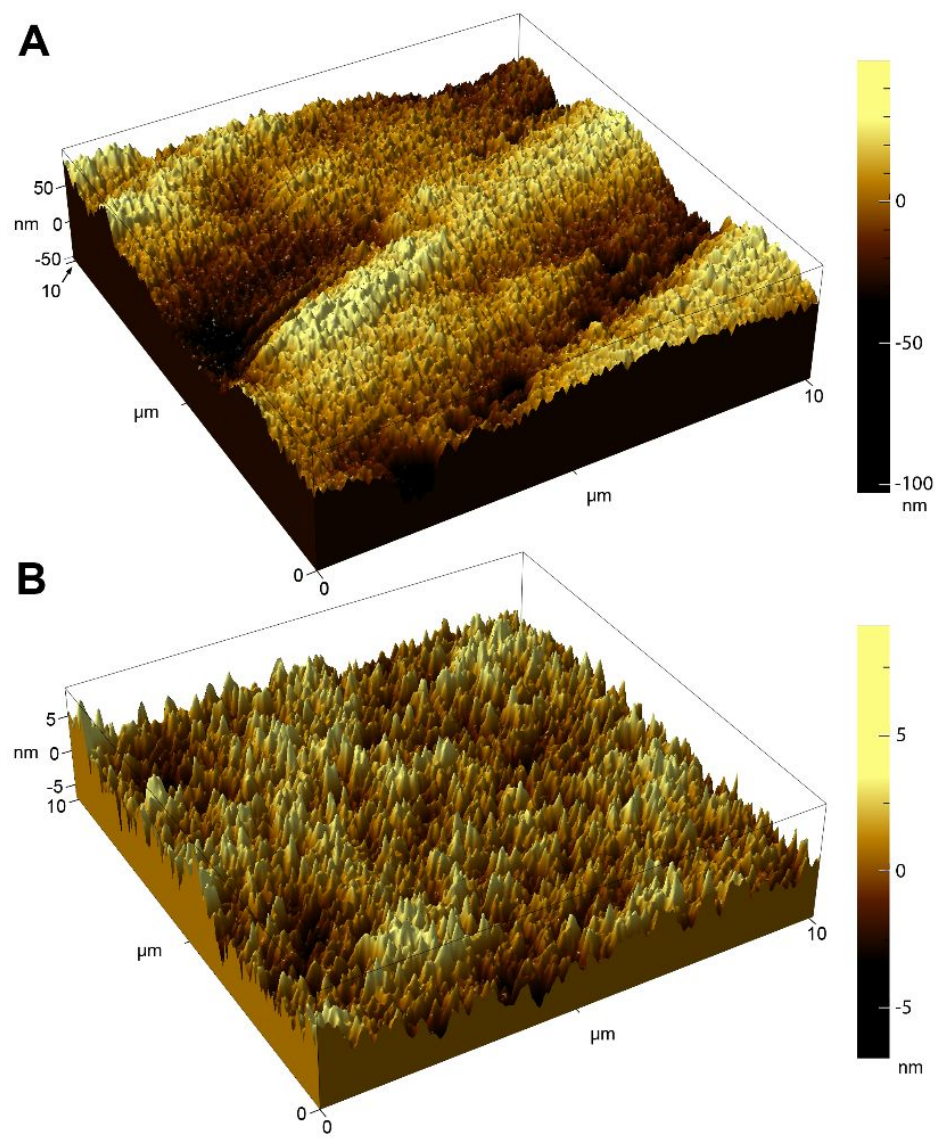

Fig. S5. AFM images of representative $10 \times 10 \mu \mathrm{m}^{2}$ fragments of the native (A) and the smoothed (B) cellulose membrane surface obtained using a SmartSPM ${ }^{\mathrm{TM}}-1000$ atomic force microscope (AIST-NT, Co, Moscow, Russia). The tapping mode with a resonance frequency of $100-150 \mathrm{kHz}$ was used in scanning experiments. 
Table S1. Roughness of native and smoothed cellulose membranes. The root mean square (RMS) roughness was measured using Gwyddion 2.45 software over $10 \mu \mathrm{m}$ regions. Scans \#1, \#2 and \#3 were obtained from the same sample at a $3 \mathrm{~mm}$ distance from each other. Run 1 and run 2 denote two independent membrane smoothing experiments.

\begin{tabular}{|l|l|l|l|l|}
\hline \multirow{2}{*}{$\begin{array}{l}\text { Scan } \\
\text { number }\end{array}$} & \multicolumn{4}{|l|}{ RMS roughness, nm } \\
\cline { 2 - 5 } & Native & Smoothed, run 1 & Smoothed, run 2 & $\begin{array}{l}\text { Smoothed, run 2 after } \\
\text { 2 months storage at } \\
\text { room temperature }\end{array}$ \\
\hline$\# 1$ & 38 & & & 2,0 \\
\hline$\# 2$ & 51 & 1,9 & 2,4 & 2,3 \\
\hline$\# 3$ & 43 & 2,0 & 2,6 & 1,7 \\
\hline
\end{tabular}

\section{Measuring the efficiency of protein immobilization}

The chemical scheme of cellulose surface modification and subsequent protein immobilization is shown in Fig. S6. The efficiency of this immobilization technique was demonstrated in a series of control experiments.

A solution of biotin-labelled BSA was spotted onto the membrane surface to produce a given surface density of a protein. A dialysis membrane was treated in radio frequency plasma discharge for 15-20 s as described earlier [S5]. A $3 \times 3 \mathrm{~mm}^{2}$ piece of the smoothed membrane (either untreated, azidophenyl-modified, or plasma-treated) was covered with $10 \mu \mathrm{L}$ of $0.01-1.0$ $\mathrm{ng} / \mathrm{mL}$ of b-BSA solution in Py-borate buffer. Untreated and plasma-treated membranes were incubated for $5 \mathrm{~min}$, whereas an azidophenyl-modified membrane was illuminated with a UV lamp (365 nm, VL-215.LC, Vilber, France) for 10 or $30 \mathrm{~s}$ at a distance of $3 \mathrm{~cm}$. Plasma-treated membrane was then immersed in a solution of $1 \%$ sodium borohydride in $200 \mathrm{mM}$ ethanolamine, $\mathrm{pH} 8.5$, for $1 \mathrm{~h}$. After the immobilization, the membrane was decorated with streptavidin-coated magnetic beads. The observed bead density values are shown in Fig. S7. If no protein was deposited onto a membrane, the beads density was $<100$ per $\mathrm{mm}^{2}$. Thus, the untreated smoothed cellulose membrane, as well as azidophenyl-modified one, has negligible nonspecific adhesion to magnetic beads. It is also clear that $10 \mathrm{~s}$ UV illumination is insufficient to bind all deposited proteins. At the same time, after $30 \mathrm{~s}$ of illumination, the photoactivated membrane binds proteins with the same efficiency as a plasma-activated membrane used previously for microarray 
fabrication [S5]. The adsorption of proteins onto the unmodified cellulose surface was expectedly low. However, as shown in the main text (Fig. 3C), at high analyte densities, the magnetic beads detection lacks linearity, and a significant analyte density increase can lead only to a small increase in the beads density. Thus, it cannot be concluded from the Fig. S7 that UV-induced immobilization is only one order of magnitude better than immobilization on an untreated membrane. In order to quantitively measure the binding capacity of an azidophenyl-modified membrane, $1 \mathrm{~cm}^{2}$ pieces of a wet membrane were covered with solutions of 0-1200 ng of Cy5labeled streptavidin in $3 \mu \mathrm{L}$ of water, covered with a piece of wet dialysis membrane and illuminated with a UV lamp for $30 \mathrm{~s}$ at a distance of $3 \mathrm{~cm}$. Thus, the thickness of a layer of the protein solution was $h=3 \mathrm{~mm}^{3} / 100 \mathrm{~mm}^{2}=0.03 \mathrm{~mm}$, and the characteristic diffusion time for this distance is $t=h^{2} / 2 D<5 \mathrm{~s}$, therefore, all protein molecules could reach the surface during UV illumination. The same is true for proteins located in the gap between touching membranes, whose height is much lower than $0.03 \mathrm{~mm}$. After washing $3 \times 1 \mathrm{~min}$ with PBS-T, samples were imaged using Typhoon FLA 9500 imager (GE Healthcare). The resulting fluorescence images are shown in Fig S8A, whereas their quantitation is presented in Fig. S8B. Saturation of the surface was achieved at a protein density of $300 \mathrm{ng} / \mathrm{cm}^{2}$. Considering the molar mass of streptavidin of $60 \mathrm{kDa}$ and the size of its molecule of $5 \mathrm{~nm}$ [S6], the dense monolayer contains $400 \mathrm{ng}$ of streptavidin per $\mathrm{cm}^{2}$. Thus, the high immobilization efficiency was confirmed, since the entire surface became occupied with protein during UV irradiation. At the same time, as follows from Fig. S8, protein binding on the native membrane, as well as on a functionalized but not irradiated membrane, was at least 2 orders of magnitude lower than in the case of photoimmobilization. Finally, after azidophenyl functionalization, the smoothed membrane had the same immobilization efficiency as the native one (the fluorescence intensity did not differ by more than $10 \%$ for each density of the immobilized protein). Obviously, the chemical nature of the surface remains unchanged, when the cellulose layer is deposited on the cellulose membrane.

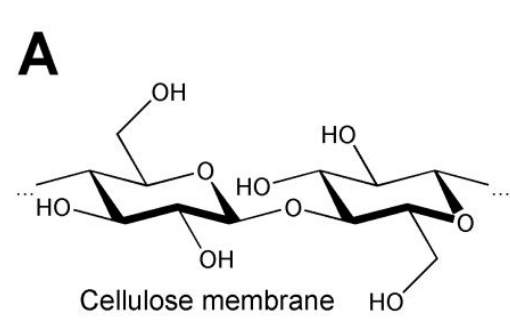

B<smiles>Cc1ccc(N)cc1</smiles>
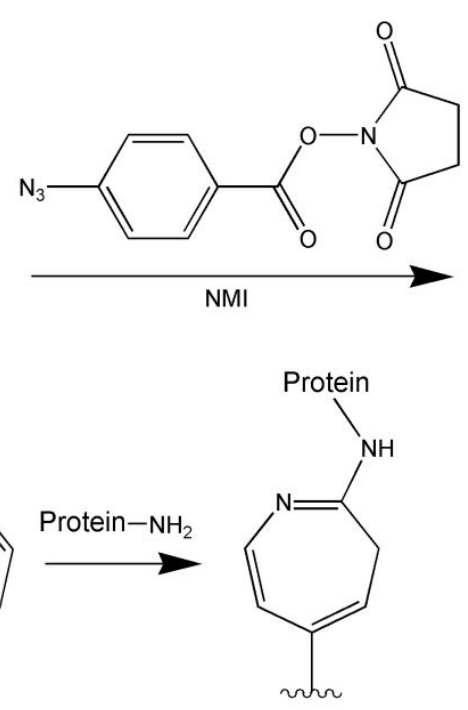

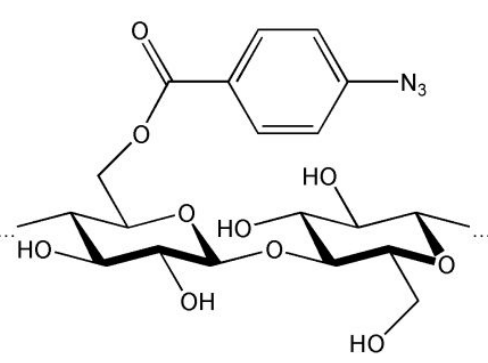


Fig. S6. The chemical schemes of (A) modification of cellulose surface with azidophenyl groups and (B) photochemical immobilization of proteins.

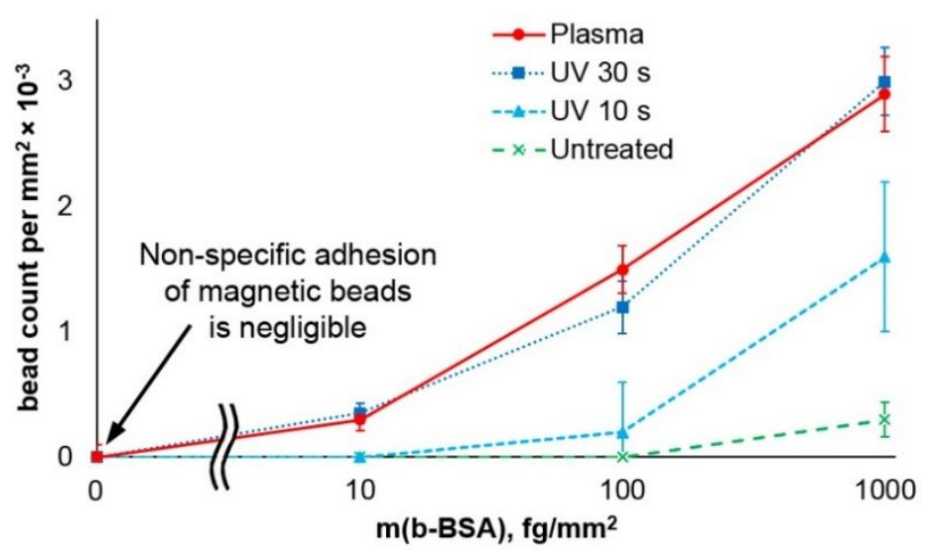

Fig. S7. The surface density of magnetic beads as a function of b-BSA mass per unit area obtained by different methods of protein immobilization. Error bars correspond to $2.5 \times \mathrm{STD}$.



Fig. S8. Measurement of the photochemical immobilization efficiency of an azidophenyl-modified cellulose membrane. (A) Fluorescence images of the membrane obtained with the indicated protein densities (in $\mathrm{ng} / \mathrm{cm}^{2}$ ). "400-1" denotes a native membrane, "400-1" denotes an 
azidophenyl-modified membrane without UV illumination, both covered with $400 \mathrm{ng} / \mathrm{cm}^{2}$ of Cy5streptavidin. (B) Fluorescence intensity as a function of Cy5-streptavin mass per unit area. Error bars correspond to $2.5 \times \mathrm{STD}$.

\section{Comparing the sensitivity of different detection techniques}

After electrophoresis of known amounts of IL-1 $\beta$ and photoimmobilization of the analyte, the membrane was incubated in $1 \mu \mathrm{g} / \mathrm{ml}$ solution of biotinylated anti-IL-1 $\beta$ antibodies in PBS-T containing $1 \%$ BSA for $1 \mathrm{~h}$, washed $3 \times 1$ min with PBS-T and used for signal detection:

1) by scanning with streptavidin coated magnetic beads, as described in the main text; the signal values were within 1.5 standard deviations from the corresponding values obtained with anti-IL-1 $\beta$-coated magnetic beads (Fig. 3C, green curve); the LOD value was also the same $-0.3 \mathrm{fg}$;

2) by incubation in a $1 \mu \mathrm{g} / \mathrm{mL}$ solution of Cy5-streptavidin (Sigma) in PBS-T containing 1\% BSA for $1 \mathrm{~h}$, washing $3 \times 1$ min with PBS-T and subsequent imaging; the LOD value was $10 \mathrm{pg}$;

3) by incubation in a $1 \mu \mathrm{g} / \mathrm{mL}$ solution of streptavidin-horseradish peroxidase conjugate (Sigma) in PBS-T containing 1\% BSA for $1 \mathrm{~h}$, washing $2 \times 1$ min with PBS-T once with PBS, soaking in ECL solution for 1.5 min and subsequent imaging; the LOD value was 1 pg.

An ECL solution was prepared immediately before use by adding $3 \mu \mathrm{L}$ of $30 \% \mathrm{H}_{2} \mathrm{O}_{2}, 50$ $\mu \mathrm{L}$ of $250 \mathrm{mM}$ luminol in dimethyl sulfoxide and $22 \mu \mathrm{L}$ of $90 \mathrm{mM}$ p-coumaric acid in dimethyl sulfoxide to $10 \mathrm{~mL}$ of 0.1 M Tris, pH 8.6 [S7]. Typhoon FLA 9500 (GE Healthcare) was used for imaging. The results of fluorescent and chemiluminescent detection are shown in Figs. S9 and S10, respectively. 


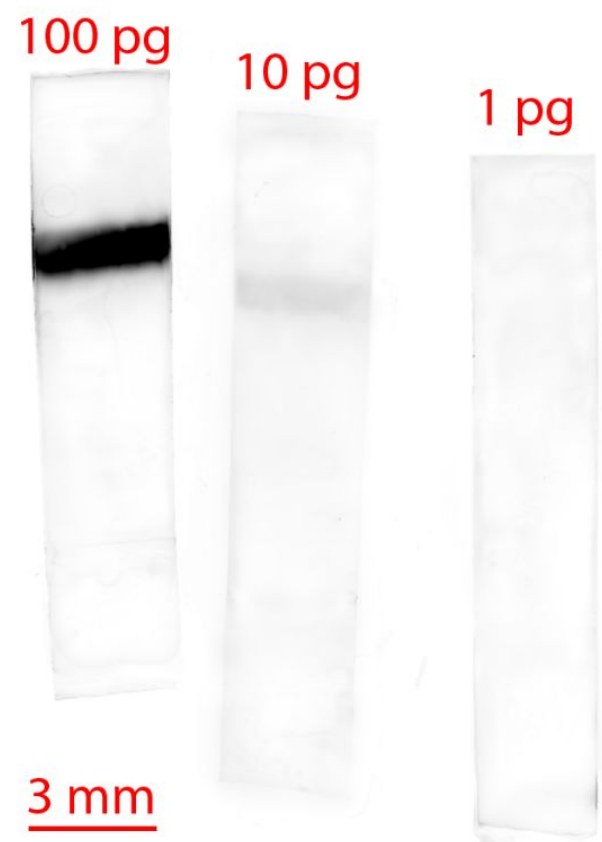

Fig. S9. Immunoblotting with fluorescent detection. See details in text.

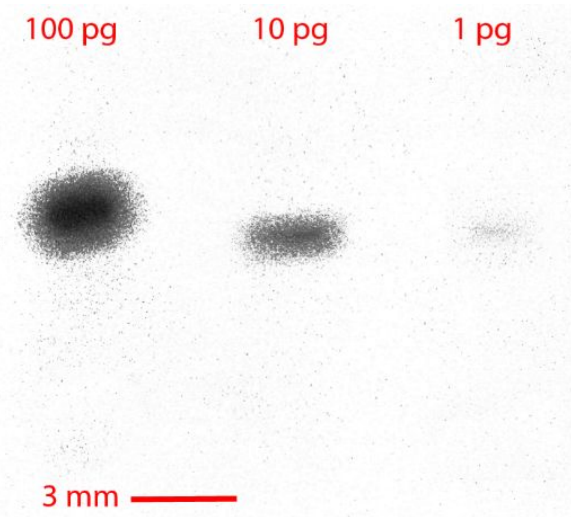

Fig. S10. Immunoblotting with chemiluminescent detection. See details in text.

\section{The sensitivity of traditional dot-blotting of IL-1ß}

Drops of an IL-1 $\beta$ solution (containing a known amount of analyte in a volume of $1 \mu \mathrm{L}$ ) were spotted onto a nitrocellulose membrane (Amersham Protran), incubated for 5 min, blocked with $3 \%$ fat-free dry milk in PBS-T, washed $3 \times 5$ min with PBS-T, incubated in a $1 \mu \mathrm{g} / \mathrm{mL}$ solution of biotinylated anti-IL-1 $\beta$ antibodies in PBS-T containing 3\% fat-free dry milk for $1 \mathrm{~h}$, washed $3 \times 5$ min with PBS-T, incubated $1 \mu \mathrm{g} / \mathrm{mL}$ streptavidin-horseradish peroxidase conjugate solution containing 3\% fat-free dry milk for $1 \mathrm{~h}$, washed with PBS-T for $3 \times 5 \mathrm{~min}$ and once with PBS. The membrane was then soaked in an ECL solution for $1.5 \mathrm{~min}$ and imaged using GE Healthcare Typhoon FLA 9500 imager. An ECL solution was prepared immediately before use by adding 3 $\mu \mathrm{L}$ of $30 \% \mathrm{H}_{2} \mathrm{O}_{2}, 50 \mu \mathrm{L}$ of $250 \mathrm{mM}$ luminol in dimethyl sulfoxide and $22 \mu \mathrm{L}$ of $90 \mathrm{mM}$-coumaric acid in dimethyl sulfoxide to $10 \mathrm{~mL}$ of 0.1 M Tris, $\mathrm{pH} 8.6$ [S7]. The results are shown in Fig. S11. 


\section{$1 \mathrm{~mm}$}

Fig. S11. Conventional dot blot of IL-1 $\beta$ with chemiluminescent detection. See details in text.

Removing magnetic beads from the surface is described in Fig. S12.

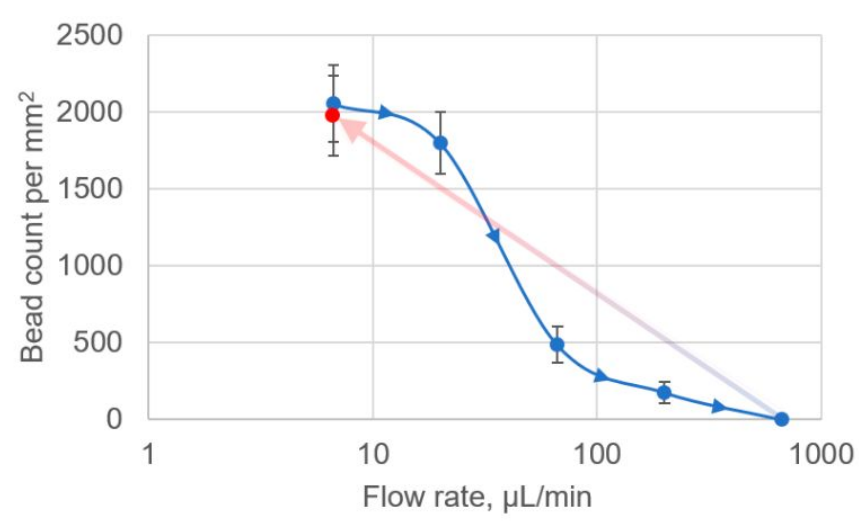

Fig. S12. Removing magnetic beads from the surface by the shear flow. An exhaled breath sample from a healthy volunteer was assayed using anti-IgA-coated magnetic beads. After the signal was accumulated at a flow rate of $7 \mu \mathrm{L} / \mathrm{min}$, the rate was stepwise increased to $670 \mu \mathrm{L} / \mathrm{min}$, at which $100 \%$ of initially bound beads were blown out. Each flow rate was applied for $10 \mathrm{~s}$. The red marker represents the signal obtained by re-scanning the same sample after complete removal of the beads at a common flow rate of $7 \mu \mathrm{L} / \mathrm{min}$.

\section{References}

S1. Ware, B. R.; Flygare, W. H. The simultaneous measurement of the electrophoretic mobility and diffusion coefficient in bovine serum albumin solutions by light scattering. Chem. Phys. Lett. 1971, 12, 81-85.

S2. Shmidt, J. L.; Cheh, H. Y. Electrophoresis along a Semipermeable Membrane Surface. Separation Science and Technology. 1992, 27, 11-27.

S3. Morozov, V. N.; Evanskey, M.; Tan, Y. K.; Shaffer, D.; Bailey, C.; Morozova, T. Y. Ultrafiltration membrane for electrophoretic capturing of pathogens for AFM imaging. Langmuir. 2006, 22, 1742-1748. 
S4. Aulin, C.; Ahola, S.; Josefsson, P.; Nishino, T.; Hirose, Y.; Osterberg, M.; Wågberg, L. Nanoscale cellulose films with different crystallinities and mesostructures-their surfaceproperties and interaction with water. Langmuir. 2009, 25, 7675-7685.

S5. Shlyapnikov, Y. M.; Shlyapnikova, E. A.; Simonova, M. A.; Shepelyakovskaya, A. O.; Brovko, F. A.; Komaleva, R. L.; Grishin, E. V.; Morozov V. N. Rapid simultaneous ultrasensitive immunodetection of five bacterial toxins. Anal. Chem. 2012, 84, 5596-5603.

S6. VanOss, C. J.; Giese, R. F.; Bronson, M.; Docoslis, A.; Edwards, P.; Ruyechan, W. T. Macroscopic-scale surface properties of streptavidin and their influence on aspecific interactions between streptavidin and dissolved biopolymers. Colloids Surf. 2003, 30, 25-36.

S7. Mruk, D. D.; Cheng, C. Y. Enhanced chemiluminescence (ECL) for routine immunoblotting: An inexpensive alternative to commercially available kits. Spermatogenesis. 2011, 1, 121-122. 